\title{
DUAL-COMPRESSION BASED MODEL USING THE ACTIVE OBJECT DETECTION MODEL
}

\author{
HARDEEP KAUR ${ }^{1} \&$ DAMANPREET KAUR ${ }^{2}$ \\ ${ }^{1}$ Research Scholar, Chandigarh University, Gharuan, India \\ ${ }^{2}$ Assistant Professor, Chandigarh University, Gharuan, India
}

\begin{abstract}
Objectives

The major objective of this image compression model is to retain the maximum quality of the main object in focus in the given image. Methods/Statistical analysis: The efficient object detection and localization algorithm has been implemented under this research, for the demarcation of the image zone containing the valuable information. The remaining image contents are compressed with the higher compression ratio based model, and the demarked zone will be compressed with maximum quality preservation method. Findings: The major results are collected in the form of image quality parameters (PSNR, MSE) and compression quality parameters (Compression Ratio) are the taken as the major performance measures. Application/Improvements: The proposed model has been primarily improved for the purpose of image quality improvement under the video conferencing models.

KEYWORDS: Active Object Detection, Efficient Compression, Dual Compression Model, Hybrid Compression, Viable Compression \& Robust Compression
\end{abstract}

Received: Jul 07, 2017; Accepted: Jul 27, 2017; Published: Aug 01, 2017; Paper Id.: IJCSEITRAUG201710

\section{INTRODUCTION}

The image size reduction is covered under the compression models designed for the 2-D, 3-D or N-D matrices, to store or transmit the images more efficiently over the given communication channel ${ }^{1}$. The image size was reduced in the percentage ranging from 1-2\% to 50-60\%, varying from the compression model to compression model $^{1-2}$. The small sized image matrices are always easier to transmit over the communication channels and easier to store on the local hard drive. The compression models usually utilizes the following

- Temporal: The temporal methods are based upon the frequency based encoding which evaluates the frequency or intensity of the image pixels for the purpose of image compression ${ }^{3}$.

- Spatial: The location based or overall image compression using the straight compression method which forcibly compresses all pixels in the image matrix ${ }^{4}$.

- Spectral: The compression model based upon the correlation and luminescence based components are utilizes for the compression of the given image matrix 5 .

- Psycho-Visual: The compilation of the image compression method based upon the object detection or object localization based upon the human visual method for the compression of the image falls under this section ${ }^{6}$. 
Image compression is performed using the various algorithms, which are designed for the rearrangement and reorganization of the pixel level information to reduce the storage size on the disk ${ }^{7-8}$. All of the compression models are utilized for the encoding of the image data bytes or pixels to group them together to reduce the overall size of image matrix is used to reduce the size of image data by fewer bytes, fewer Mbytes or more ${ }^{9}$. The image encoding and decoding mechanisms ${ }^{10}$ are utilized for the compression and decompression ${ }^{11}$ practices by archiving ${ }^{12}$, restructuring ${ }^{13}$ and other variants to transform the image matrix into the smaller sizes are the major reasons behind the image compression models ${ }^{14}$.

\section{EXPERIMENTAL DESIGN}

The main goal of the dual compression based model was to propose the robust compression in the images or video frames. The proposed model consisted of the object detection, object compression and surrounding compression models. The proposed model engulfs the compression problems with design based upon the DCT, DFT and DWT models. For images, the JPEG images are taken into account as it preferred DWT over DCT or DFT. [12, 13] In DFT execution, time is lower and it provides lower compression as compare to the other techniques. In DCT, it is a simple compression algorithm, because computation count in this algorithm is limited, hence provides lower compression ratio. DWT on the other hand, is complex and computation count is very high and it provides higher compression ratio as compared to later two and also proven to be more effective.

Under the wavelet based methods, the image is first derived into the wavelet components such as approximation coefficient, diagonal coefficient, horizontal coefficient and vertical coefficient, known as $\mathrm{CA}, \mathrm{CD}, \mathrm{CH}$ and $\mathrm{CV}$ respectively. The DWT based wavelet model by using the HAAR variant is prominently utilized for the higher order compression and lowering the elapsed time. Finally, the decision is made to use DWT for its effectiveness and robustness over DCT and DFT. [10,11]

\section{Input Data}

The input data image undergoes the object detection and demarcation model in the very first step of segmentation. The input image is analyzed for the position of the objects, and which are further demarked and the position of the corner points in returned to the main program. The input data is further passed to the next step for the compression.

\section{Apply Run Length Encoding}

Then the run length encoding is applied over the detected object region after its extraction from the image using the corner points defined by the object detection method in the first step. The object region is extracted and compressed with the run-length encoding using the following method. 


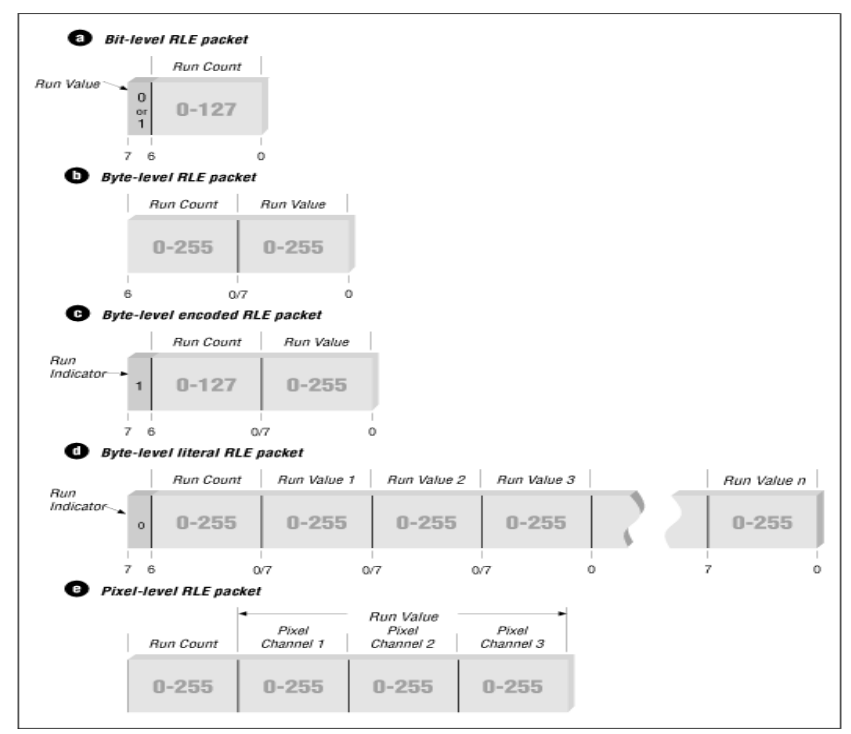

Figure 1

\section{Apply Huffman Encoding}

Then the Huffman encoding is applied over the extracted objects for the achievement of higher level compression. The Huffman encoding method was originally developed by the David A. Huffman. The Huffman model consists of the arrangement of the variable-length codes for the symbols using the pre-defined symbol to code mapping database.

Input

Alphabet $X=\left\{x_{1}, x_{2}, x_{3} \ldots x_{N}\right\}$, for vector of length $(N)$ to map the alphabets in the alphabet list

Set Weight $=\left\{\mathrm{w}_{1}, \mathrm{w}_{2}, \mathrm{w}_{3} \ldots \mathrm{w}_{\mathrm{N}}\right\}$, can be defined as the list of consisted weights in the list of the symbols to determine the positive and negative weights on the entitled list.

\section{Output}

Encode $E(X$, Weight $)=\left\{e_{1}, e_{2}, e_{3} \ldots e_{N}\right\}$, which converts the target symbols to the mapped weights in the given mapping list (also called codebook).

\section{Goal}

Let $\operatorname{LE}(\mathrm{E})=\sum_{i=1}^{n} w(i) *$ lengt $\square\{c(i)\}$, which eventually combines the converted codes into the output codebook using the condition $\mathrm{L}(\mathrm{C}) \leq \mathrm{L}(\mathrm{T})$ for all code in the codebook list $\mathrm{E}(\mathrm{X}$, Weight).

\section{Apply Discrete Wavelet Compression}

The remaining image parts are compressed by using the wavelet compression using the DWT (Discrete Wavelet Transform) model. The wavelet model derives the input image matrix to the low level coefficient matrices, which are also known as the sub-bands or derivatives of the image matrix. Out of all image coefficients, the absolute coefficient solely is utilized to reconstruct the original image, which means that the absolute coefficient matrix can be taken as the compression image portion. The equation below describes the working of the DWT: 


$$
y[n]=(x * g)[n]=\sum_{k=-\infty}^{\infty} x[k] g[n-k]
$$

The DWT model computes the high and low frequency components using the set of following equations:

$$
\begin{aligned}
& y_{\text {low }}[n]=\sum_{k=-\infty}^{\infty} x[k] h[2 n-k] \\
& y_{\text {high }[n]}=\sum_{k=-\infty}^{\infty} x[k] g[2 n-k]
\end{aligned}
$$

\section{RESULTS ANALYSIS}

A standard image dataset has been utilized under this research, which has been derived from the Prof. James Wang's dataset of image collected from the various categories. This dataset is known for the Semantics-sensitive image practices on the image matrices for the content based image retrieval (CBIR) models, compression \& decompression ( $\operatorname{codec})$ models, etc. The semi-image dataset has been derived from the Prof. Wang's dataset, which consists of total 52 images for the compression practices. The proposed model has been tested on the given dataset for the evaluation of the proposed model on the basis of the various performance parameters such as peak signal to noise ratio (PSNR), mean squared error (MSE) and compression ratio (CR). The image dataset is containing the five types of images, which include the types of the images such as natural scenes with regular color illumination, ancient building or monument images, standard images like Lena, etc. The following snapshot shows the workflow of the compression based algorithm:

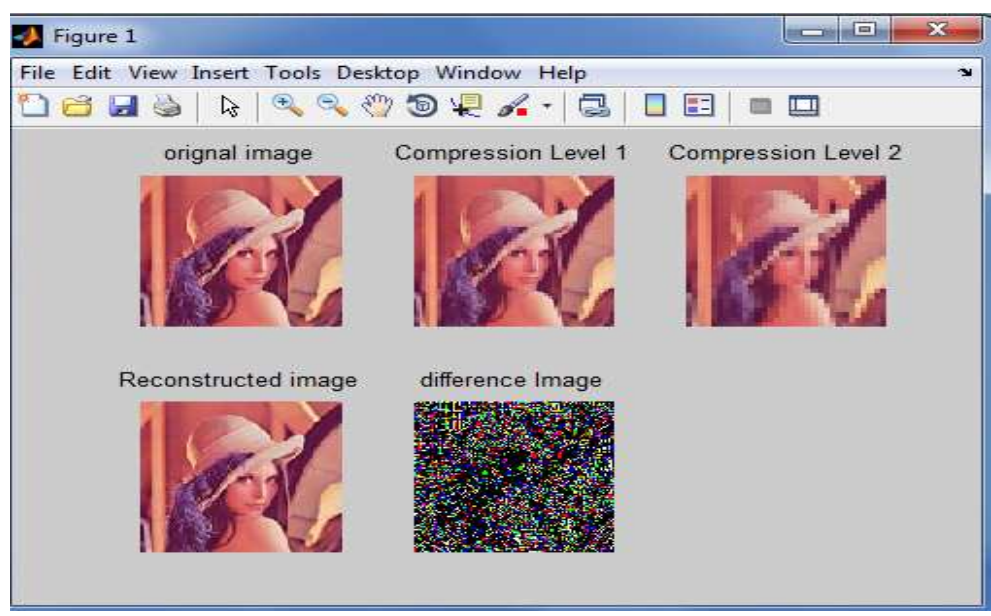

Figure 5.1: Compressed Image

Elapsed time or the time cost is the parameter, under which we track the time taken for the target procedure (compression \& decompression time in this case) and measured in seconds. The value of elapsed time has been measured in the rotation under our proposed model for each and every tested image. The figure 5.2 shows the results obtained for elapsed time parameter over the different images from the given dataset. 


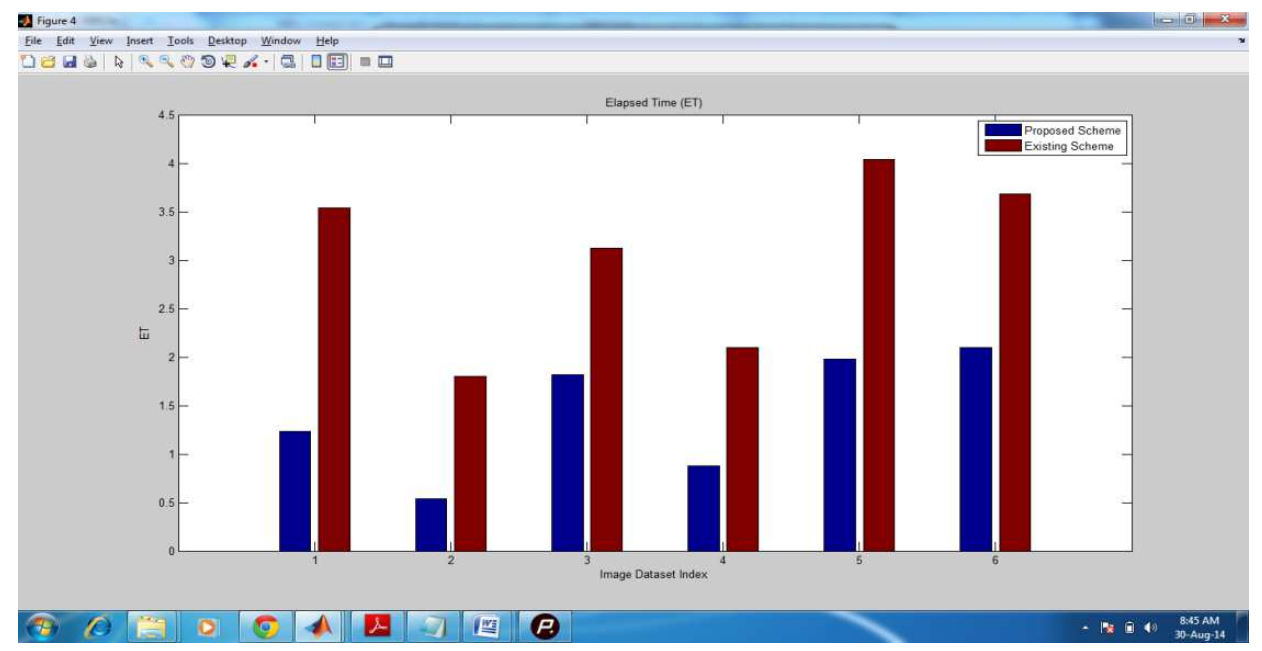

Figure 5.2: Parameter of Elapsed Time Measured B/W Existing and Proposed Model

The peak signal to noise ratio (PSNR) is the parameter utilized for the measurement of the quality of the image matrix in 2-D or 3-D image and between the original and the processed image. The PSNR can vary between any range (usually ranging from 0-150, as per its known from the literature study), which elaborates the performance of the analysis model. The higher value of PSNR indicates the better performance of the model and vice versa. The following image (Figure 5.3) shows the results for the PSNR between the proposed and existing models:

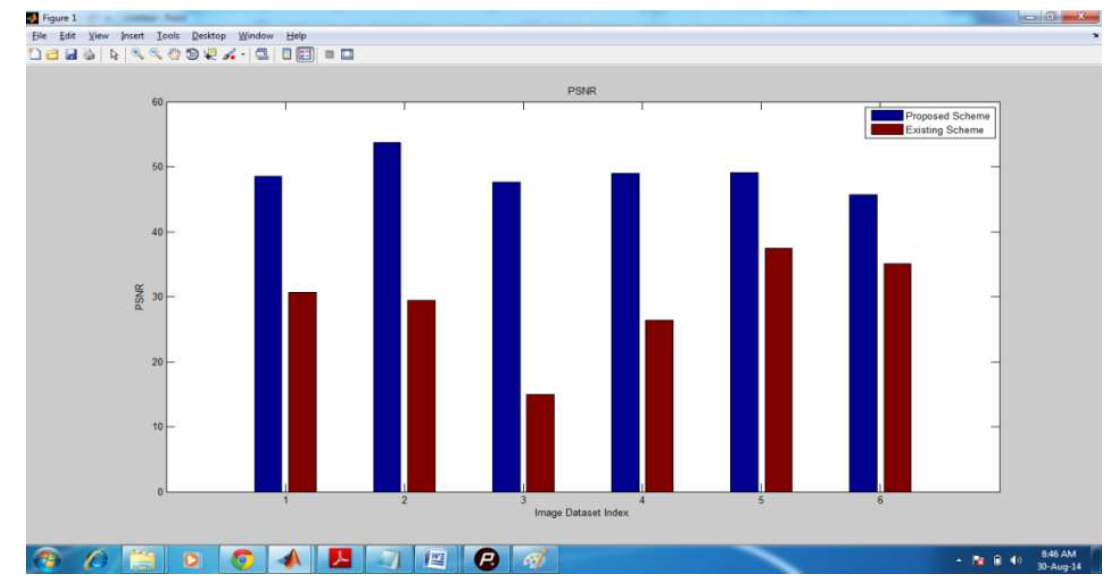

Figure 5.3: Parameter of PSNR Measured B/W Existing and Proposed Model

The mean squared error (MSE) computes the number of error values or the values, which are changed during the processing of the proposed compression model and further divided by the total of the values in the given image matrix. The proposed model and existing models are compared on the basis of the compression quality based performance on the scale of MSE between the proposed and existing models under this research. 


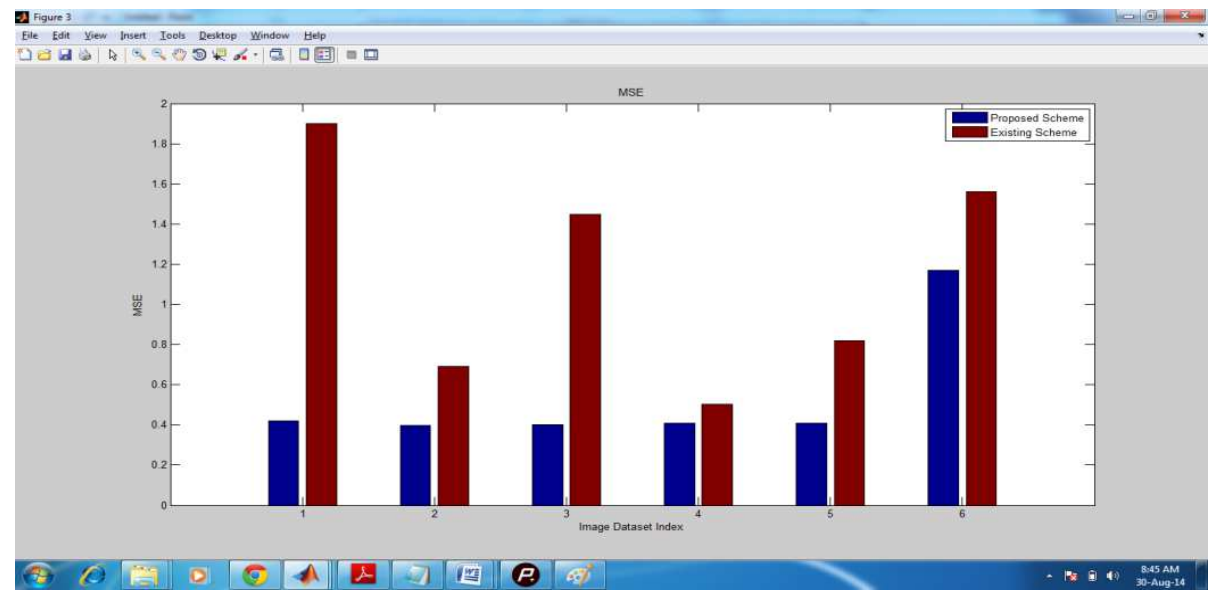

Figure 5.4: Parameter of Mse Measured B/W Existing and Proposed Model

The compression ratio (CR) is the parameter of size of the image matrix changed during the image processing practices. The compression ratio is computed by obtaining the size of the image matrix after the processing and dividing it by the size of the original image, which is further subtracted from 100 to obtain the percentage of change in the given image matrix.

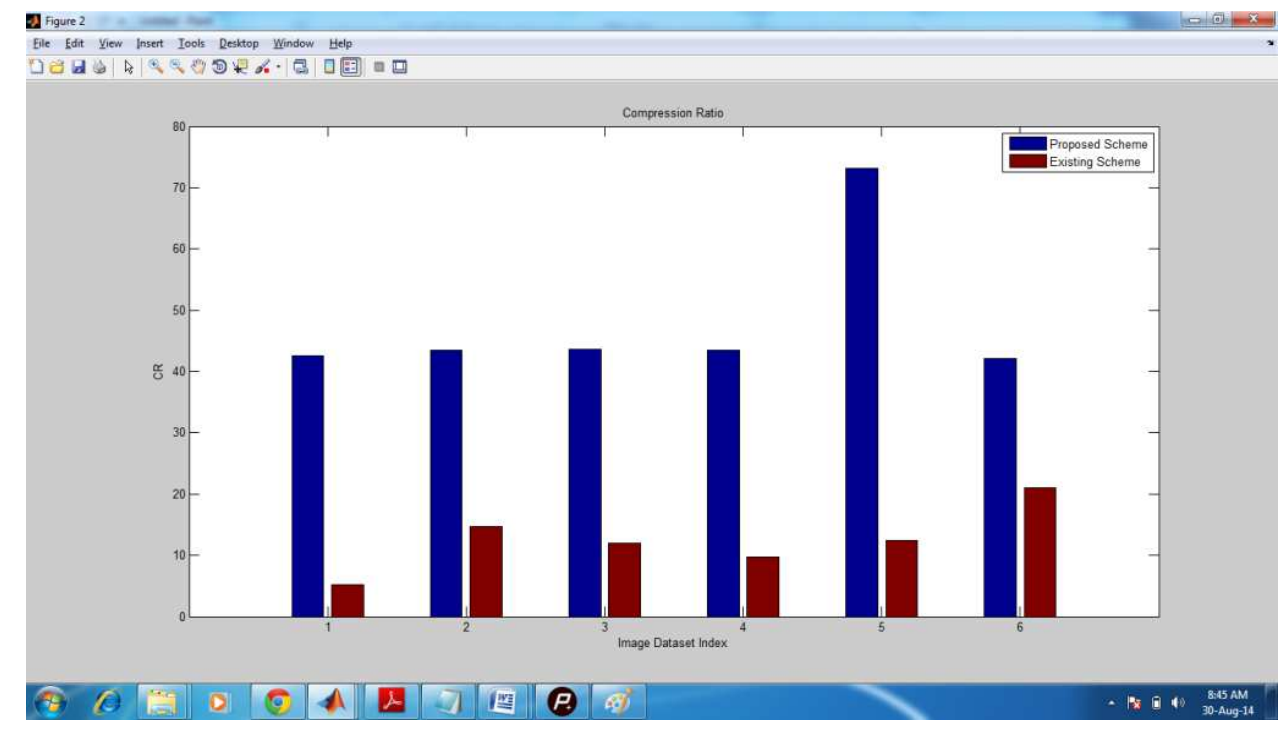

Figure 5.5: Parameter of CR Measured B/W Existing and Proposed Model

Table 5.1: Comparative Analysis Based Upon PSNR B/W Existing \& Proposed Techniques

\begin{tabular}{|l|c|c|c|}
\hline \multirow{2}{*}{ Image Group in Dataset } & \multirow{2}{*}{ Data Set Index } & \multicolumn{2}{|c|}{ PSNR } \\
\cline { 3 - 4 } & & Proposed & Existing \\
\hline & & & \\
\hline & & & \\
& & & \\
\hline
\end{tabular}




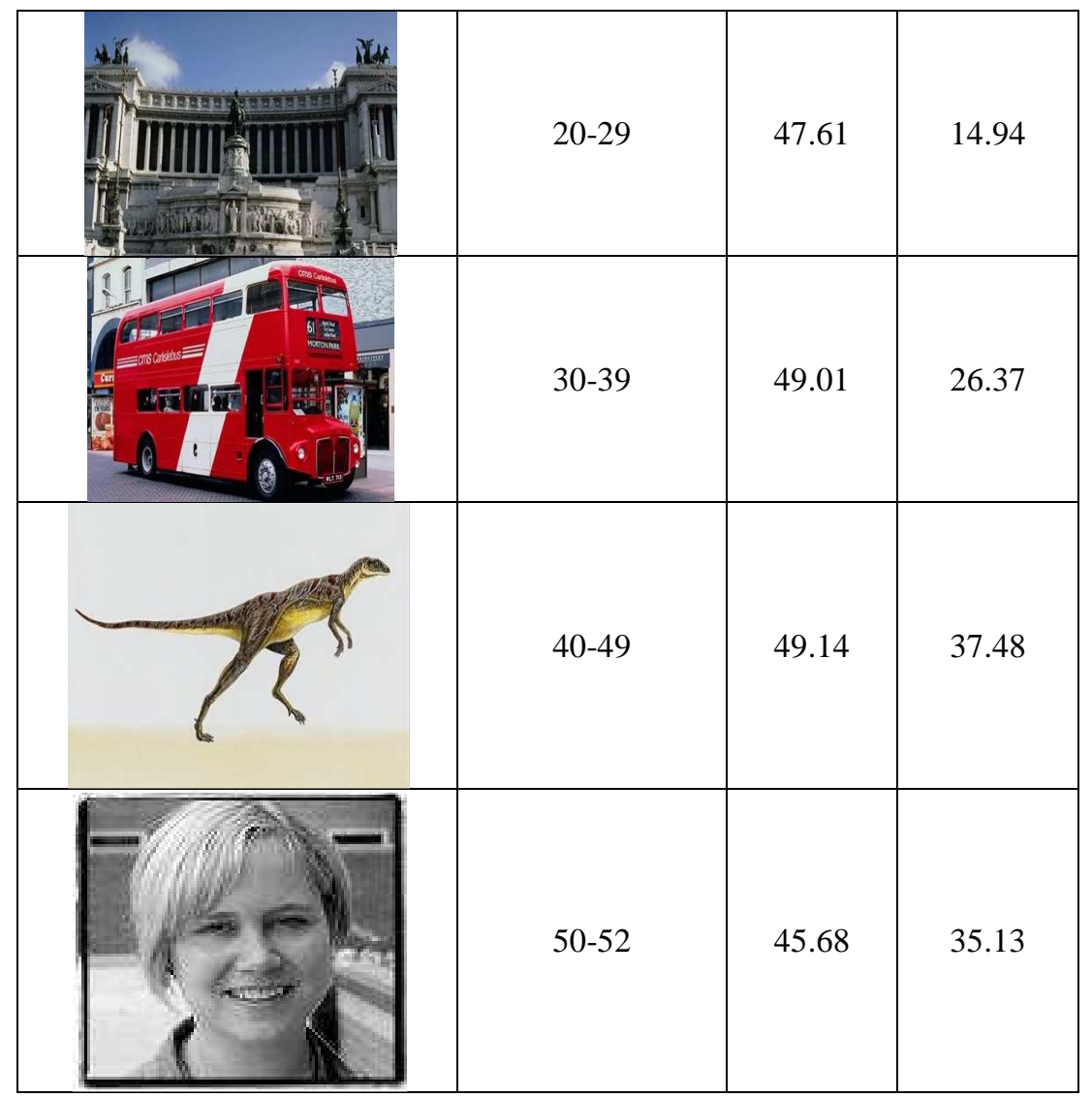

Table 5.2: Comparative Analysis Based Upon CR B/W Existing \& Proposed Techniques

\begin{tabular}{|c|c|c|c|}
\hline Image Group in Dataset & Data Set Index & \multicolumn{2}{|c|}{ Compression Ratio (CR) } \\
\cline { 2 - 4 } & & Proposed & Existing \\
\hline & $0-7$ & 42.58 & 5.237552 \\
\hline & & & \\
\hline & & & \\
\hline & & & \\
\hline & & & \\
\hline & & & \\
\hline & & & \\
\hline
\end{tabular}




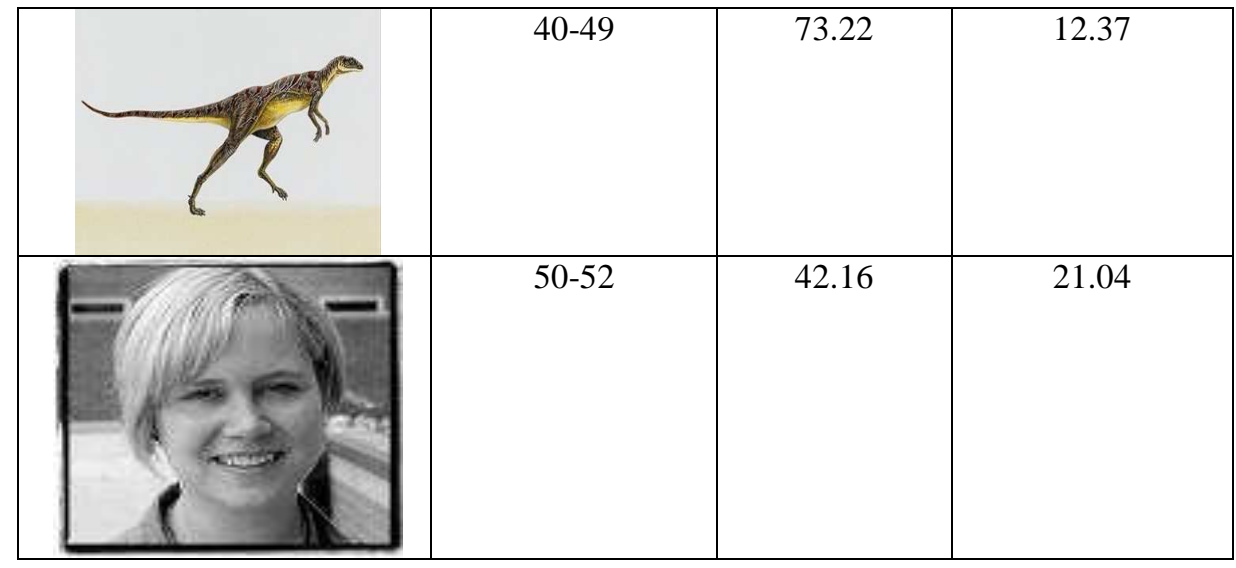

Table 5.3: Comparative Analysis Based Upon MSE B/W Existing \& Proposed Techniques

\begin{tabular}{|c|c|c|c|}
\hline Image Group in Dataset & Data Set Index & \multicolumn{2}{|c|}{ MSE } \\
\hline & $0-7$ & 0.420 & 1.90 \\
\hline & 8-19 & 0.398 & 0.69 \\
\hline & $20-29$ & 0.40 & 1.45 \\
\hline & $30-39$ & 0.41 & 0.504 \\
\hline & $40-49$ & 0.408 & 0.819 \\
\hline & $50-52$ & 1.168 & 1.56 \\
\hline & & & \\
\hline
\end{tabular}


Table 5.4: Comparative Analysis Based Upon Et B/W Existing \& Proposed Techniques

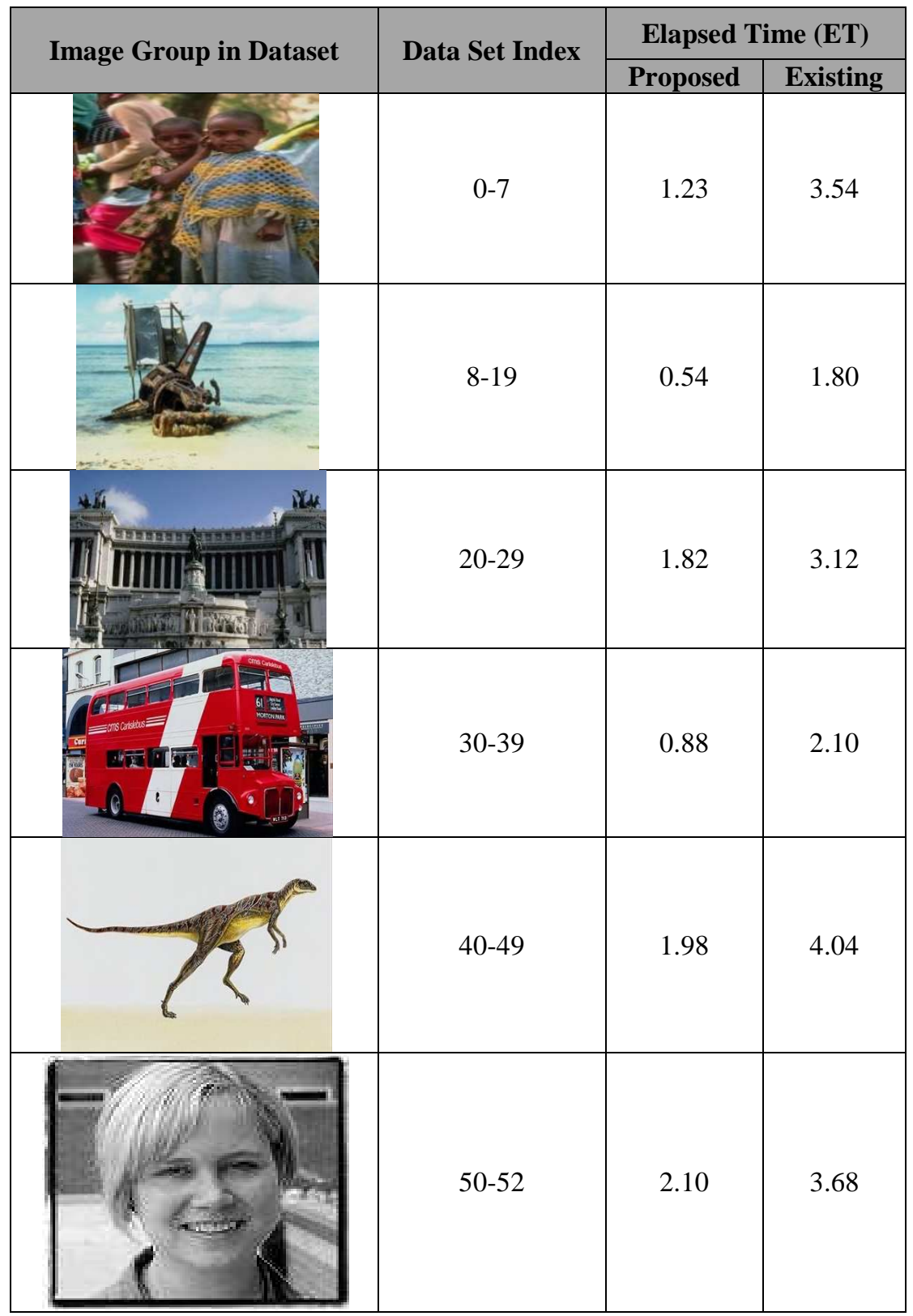

\section{CONCLUSIONS}

The proposed model has been designed for the dual-layered compression of the image data after extracting the important or objects in main focus to compress with the lossless image compression models, whereas the remaining image matrix is compressed by using the higher compression model using the lossy compression model. The proposed model utilizes the combination of DWT and SVD for the lossy compression which has achieved nearly 70-80\% of compression of the image matrix, whereas the lossless compression model based upon the Entropy and Huffman encoding achieves the compression ratio of nearly $15-20 \%$ at maximum. This model keeps the in-focus object quality in the high quality resolution and with higher pixel density, which does not interrupt the overall quality and lets the viewer to visualize the clearer objects. The performance of the proposed model has been analyzed under the performance parameters of MSE, ET, PSNR and compression ratio (CR) for its assessment against the existing models of image compression. The proposed 
model has outperformed the existing model by nearly $5-20 \%$ or above on the basis of all of the latter mentioned parameters, which ironically signifies the improved performance of the proposed model in comparison with the existing model.

\section{REFERENCES}

1. Aggarwal, Preeti. and Rani, Babita (2010), "Performance Comparison of Image Compression Using Wavelets", International Journal of Computer Science \& Communication, Vol No. 1, Issue No. 2, pp. 97-100.

2. Al-lahan, Mohammed. and El Emary, Ibrahiem M. M. (2007), "Comparative Study between Various Algorithms of Data Compression Techniques”, Proceedings of the World Congress on Engineering \& Computer Science.

3. Amrut N. Patel, Ravindra M. Patel, D. J. Shah (2013), "Performance Analysis of Wavelet Families for Image Compression", International Journal of Engineering and Innovative Technology (IJEIT) Volume 2, Issue 12.

4. Anish Kumar, M. S., Roy, Rajesh Cherian. And Gopikakumari, R. (2006), "A New Image Compression and Decompression technique based on 8x8 MRT”, GVIP Journal, Volume 6, Issue 1, July 2006.

5. Asokan, Anju, and J. P. Anita. "Burrows Wheeler Transform Based Test Vector Compression for Digital Circuits." Indian Journal of Science and Technology 9, no. 30 (2016).

6. B. B. S. Kumar and P. S. Satyanarayana (2013), "Compression and Denoising - Comparative Analysis from still Images using Wavelet Techniques", ITSI Transactions on Electrical and Electronics Engineering (ITSI-TEEE) ISSN (PRINT) : 2320 - 8945, Volume -1, Issue -6.

7. Dipalee Gupta and Siddhartha Choubey, "Discrete Wavelet Transform for Image Processing", International Journal of Emerging Technology and Advanced Engineering (ISSN 2250-2459, ISO 9001:2008 Certified Journal, Volume 4, Issue 3.

8. Lee, Geun-Ho, Sun-Woo Ko, and Soo-Tae Kwon. "A Study on the Differential Compression Method for High-Speed Transfer." Indian Journal of Science and Technology 8, no. S1 (2015): 524-527.

9. Noor HudaJa'afar et.al (2013) "Distributed Arithmetic Architecture of Discrete Wavelet Transform (DWT) with Hybrid Method"

10. Oussama GHORBEL and Walid AYEDI (2012)," Images Compression in WSN: Performance Analysis" IEEE.

11. Sandeepkaur, GaganpreetKaur and Dr. Dheerendra Singh (2013), "Comparative Analysis of Haar And Coiflet Wavelets Using Discrete Wavelet Transform In Digital Image Compression "International Journal of Engineering Research and Applications (IJERA) Vol. 3, Issue 3, pp.669-673 669

12. Singh, Indraj, and V. Sahni. "Performance and Emission Analysis of Compression Ignition Engine Coupled with Electric Generator Set used Bakain Methyl Ester as Fuel." Indian Journal of Science and Technology 9, no. 33 (2016).

13. Sivaganesan, S., and M. Chandrasekaran. "Performance and Emission Analysis of Compression Ignition Engine with Methyl Ester of Jatropha and Diesel." Indian Journal of Science and Technology 9, no. 26 (2016).

14. Srinivasan, V. "Thermal Analysis of Compression Ignition Engine Muffler and its Design Modification." Indian Journal of Science and Technology 8, no. 31 (2015). 